Pontifícia Universidade Católica $_{\text {mano }}$

Rodrigo Corrêa Torres

Avaliação de Portfolios de Contratos de Compra e Venda de Energia Elétrica: Uma Abordagem pela Teoria de Opções

Dissertação de Mestrado

Dissertação apresentada ao Programa de Pós-graduação em Engenharia Industrial da PUC-Rio como requisito parcial para obtenção do título de Mestre em Engenharia Industrial.

Orientador: Tara Keshar Nanda Baidya

Rio de Janeiro

Janeiro de 2006 
Rodrigo Corrêa Torres

\section{Avaliação de Portfolios de Contratos de Compra e Venda de Energia Elétrica: Uma Abordagem pela Teoria de Opções}

Dissertação apresentada como requisito parcial para a obtenção do grau de Mestre pelo Programa de Pós-graduação em Engenharia Industrial da PUC-Rio. Aprovada pela Comissão Examinadora abaixo assinada.

Prof. Tara Keshar Nanda Baidya

Orientador

DEI - PUC-Rio

\section{Prof. Carlos Patrício Samanez}

DEI - PUC-Rio

Prof. Leonardo Lima Gomes

Fucape - Vitória

Prof. José Eugênio Leal

Coordenador Setorial do Centro Técnico Científico - PUC-Rio 
Todos os direitos reservados. É proibida a reprodução total ou parcial do trabalho sem autorização da universidade, do autor e do orientador.

\section{Rodrigo Corrêa Torres}

Graduou-se em Engenharia Civil na Universidade Federal do Rio de Janeiro em 1999. Durante o período de graduação, estagiou em diversas empresas e foi bolsista CNPQ. Pósgraduou-se em administração na U.C. Berkeley em 2000. Trabalhou no Cepel durante quatro anos em projetos relacionados à avaliação de empreendimentos no setor elétrico brasileiro. Atualmente trabalha como analista comercial na NC Energia.

Ficha Catalográfica

Torres, Rodrigo Corrêa

Avaliação de portfolios de contratos de compra e venda de energia elétrica: uma abordagem pela teoria de opções / Rodrigo Corrêa Torres ; orientador: Tara Keshar Nanda Baidya. - Rio de Janeiro : PUC, Departamento de Engenharia Industrial, 2005.

117 f. ; $30 \mathrm{~cm}$

Dissertação (mestrado) - Pontifícia Universidade Católica do Rio de Janeiro, Departamento de Engenharia Industrial.

Inclui referências bibliográficas.

1. Engenharia industrial - Teses. 2. Avaliação de Portfolios. 3. Análise de Risco. 4. Simulação de Monte Carlo. 5. Avaliação de Opções. I. Baidya, Tara Keshar Nanda. II. Pontifícia Universidade Católica do Rio de Janeiro. Departamento de Engenharia Industrial. III. Título. 


\section{Agradecimentos}

Aos meus pais Manoel e Vera Lúcia, minha irmã Ana Paula e meu irmão Renan, pelo carinho, pela paciência e todo apoio dado durante o mestrado.

Ao meu orientador, Professor Tara Keshar Nanda Baidya e a Leonardo Lima Gomes pelo estimulo e pela colaboração neste trabalho.

Aos professores José Paulo Teixeira e Carlos Patrício Samanez pelos ensinamentos dados.

A Albert C. Geber Melo e a Fabio Rodrigo Siqueira Batista pelo incentivo dado em iniciar este programa de mestrado.

Aos amigos do Cepel, pela ajuda e amizade ao longo deste curso.

Aos amigos do mestrado, pelo companheirismo e união.

Aos funcionários do DEI, pela ajuda e amizade ao longo deste curso. 


\section{Resumo}

Torres, Rodrigo Corrêa; Baidya, Tara Keshar Nanda. Avaliação de Portfolios de Contratos de Compra e Venda de Energia Elétrica: Uma Abordagem pela Teoria de Opções. Rio de Janeiro, 2006. 117p. Dissertação de Mestrado - Departamento de Engenharia Industrial, Pontifícia Universidade Católica do Rio de Janeiro.

O Ambiente de Contratação Livre proporcionou uma continuidade do processo de livre concorrência de mercado iniciado com a reestruturação do setor elétrico em 1997. A mudança de um regime baseado em contratos de suprimento renováveis para uma estrutura baseada em preços dados por um mercado competitivo, expõe as empresas do setor elétrico brasileiro à volatilidade do mercado de eletricidade. Neste novo ambiente, as empresas devem gerenciar os riscos associados às suas operações. Devido às características singulares do setor elétrico brasileiro, o gerenciamento de risco é um grande desafio para os próximos anos. Por outro lado, com a liberdade de negociação permitida pelo segmento de comercialização de energia no Ambiente de Contratação Livre, os contratos de compra e venda de energia elétrica passaram a adaptar-se as necessidades de mercado com a incorporação de flexibilidades que viessem a mitigar os riscos com relação à demanda por energia elétrica e principalmente com relação ao preço. Dentro desse contexto, foi desenvolvido um modelo de avaliação de portfolio de contratos de compra e venda de energia elétrica, incorporando as flexibilidades inerentes a atividade de comercialização, de forma a quantificar os riscos associados a esta atividade e determinar o valor adicionado ao portfolio pelas flexibilidades. O caso estudado é fictício, mas é um exemplo típico na área de comercialização de energia elétrica dentro deste novo modelo.

\section{Palavras-chave}

Avaliação de Portfolios; Análise de Risco; Simulação de Monte Carlo; Avaliação de Opções. 


\section{Abstract}

Torres, Rodrigo Corrêa; Baydia, Tara Keshar Nanda. Portfolio Valuation of Electricity Contracts: an Options Theory Approach. Rio de Janeiro, 2006. 117p. MSc. Dissertation - Departamento de Engenharia Industrial, Pontifícia Universidade Católica do Rio de Janeiro.

The Free Contracts Environment enabled continuity of the free market competition process which started with the electric sector restructure in 1997. The shift from a regime based on renewable supply contracts to a structure based on prices established by competition exposes companies in the Brazilian electric sector to the volatility of the electricity market. In this new environment companies must manage the risks associated to the operations. The Brazilian electric sector singular features make risk management a great challenge for ensuing years. On the other hand, with free negotiation enabled by the energy trade segment within the free contracts environment, electric energy purchase and sale contracts started to adapt to the market needs incorporating flexibilities designed to face uncertainty regarding electric energy demand in general and prices in particular. Within this context, an electric energy purchase and sale portfolio valuation model was developed, incorporating the flexibilities inherent to commercialization activities, in order to quantify the risks associated with this activity and establish the value added to the portfolio by the flexibilities. The case studied is fictitious, but typical in the field of electric energy trading within this new model.

\section{Keywords}

Portfolio Valuation; Risk Analysis; Monte Carlo Simulation; Options Valuation. 


\section{Sumário}

1 INTRODUÇÃO

\section{AVALIAÇÃO DE PORTFOLIOS NA ÁREA DE COMERCIALIZAÇÃO} DE ENERGIA ELÉTRICA 20

2.1. A Nova Estrutura de Comercialização 21

2.2. Formação do Preço de Liquidação de Diferenças e do Preço de Contratos Bilaterais

2.3. Contratos de Compra e Venda de Energia Elétrica 31

2.4. Avaliação de Ativos e Gerenciamento de Risco 37

3 BASE TEÓRICA $\quad 40$

3.1. Risco e Retorno de Carteiras $\quad 40$

3.2. Métricas de Risco 43

3.2.1. Value at Risk (VaR) 44

3.2.2. Conditional Value at Risk (CVaR) 48

3.3. Opções Financeiras $\quad 50$

3.3.1. Simulação de Monte Carlo para Avaliação de Opções Européias 55

3.3.2. Teoria das Opções Aplicada ao Setor Elétrico Brasileiro 58

4 MODELAGEM MATEMÁTICA $\quad 60$

4.1. Simulação do Preço de Liquidação de Diferenças (PLD) 60

4.2. Incerteza com Relação ao Consumo de Energia 65

4.3. Cálculo do Valor Esperado do Portfolio 68

4.4. Cálculo dos Valores em Risco 74 
4.5. Cálculo do Valor Adicionado pelas Flexibilidades nos Contratos de Compra

5.1. Premissas do Exemplo Numérico

5.2. Análise de Risco e Valor do Portfolio

5.2.1. Sensibilidade sobre a Taxa de Desconto 86

5.2.2. Sensibilidade sobre a Flexibilidade dos Contratos de Compra 87

5.2.3. Sensibilidade sobre o Nível de Confiança do VaR e do CVaR 88

5.2.4. Sensibilidade sobre o Número de Simulações do MAB 89

5.2.5. Risco Volumétrico

5.3. Precificação das Flexibilidades nos Contratos de Compra de Energia Elétrica

5.3.1. Sensibilidade sobre a Taxa de Desconto

5.3.2. Sensibilidade Sobre o Preço dos Contratos de Curto Prazo 


\section{Lista de Figuras}

FIGURA 2.1 - ESTRUTURA DE COMERCIALIZAÇÃO NO SETOR ELÉTRICO BRASILEIRO 22

FIGURA 2.2 - EVOLUÇÃO DO MERCADO LIVRE NA EUROPA 22 FIGURA 2.3 - PROCESSO DE DECISÃO PARA SISTEMAS

HIDROTÉRMICOS 26

FIGURA 2.4 - FUNÇÕES DE CUSTO IMEDIATO E FUTURO X

ARMAZENAMENTO 26

FIGURA 2.5 - USO ÓTIMO DA ÁGUA EM SISTEMAS HIDROTÉRMICOS 27

FIGURA 2.6 - EVOLUÇÃO DOS PLDS 30

FIGURA 2.7 - OPÇÃO DE COMPRA E VENDA 34

FIGURA 2.8 - PORTFOLIO 34

FIGURA 2.9 - EXERCÍCIO DA OPÇÃO DE VENDA 34

FIGURA 2.10 - EXERCÍCIO DA OPÇÃO DE COMPRA 35

FIGURA 2.11 - HISTÓRICO DE CONSUMO DE ENERGIA ELÉTRICA INDÚSTRIA QUÍMICA 36

FIGURA 2.12 - TAKE OR PAY - CONTRATOS DE VENDA 37

FIGURA 2.13 - EXPOSIÇÃO DE CONTRATOS 39

FIGURA 4.1 - DISTRIBUIÇÃO DE PLDS 64

FIGURA 4.2 - MAB PARA O CONSUMO DE ENERGIA 68

FIGURA 5.1 - ÁGIO SOBRE O PLD (\%) 80

FIGURA 5.2 - PORTFOLIO DE CONTRATOS DE COMPRA E VENDA 82

FIGURA 5.3 - PROJEÇÃO DE PLDS - SUBMERCADO SUDESTE 83

FIGURA 5.4 - PROJEÇÃO DE PLDS - SUBMERCADO NORDESTE 83

FIGURA 5.5 - PROJEÇÃO DE PLDS - SUBMERCADO SUL 84

FIGURA 5.6 - RISCO E RETORNO DO PORTFOLIO - CASO BASE 85

FIGURA 5.7 - SENSIBILIDADE SOBRE A TAXA DE DESCONTO 86

FIGURA 5.8 - SENSIBILIDADE SOBRE A FLEXIBILIDADE DOS

CONTRATOS DE COMPRA - RETORNO ESPERADO 87

FIGURA 5.9 - SENSIBILIDADE SOBRE A FLEXIBILIDADE DOS

CONTRATOS DE COMPRA - MÁXIMO VAR E CVAR 87 
FIGURA 5.10 - SENSIBILIDADE SOBRE O NÍVEL DE CONFIANÇA DO VAR E DO CVAR

FIGURA 5.11 - PERFIL DE RISCO DO AGENTE PARA DIFERENTES NÍVEIS DE CONFIANÇA

FIGURA 5.12 - CONVERGÊNCIA DAS ESTIMATIVAS DE VP - DESVIO PADRÃO

FIGURA 5.13 - CONVERGÊNCIA DAS ESTIMATIVAS DE VP COEFICIENTE DE VARIAÇÃO

FIGURA 5.14 - LIMITES MÁXIMO E MÍNIMO DOS CONTRATOS DE VENDA

FIGURA 5.15 - VALOR TOTAL DAS OPÇÕES (MILHÕES R\$) 94

FIGURA 5.16 - CONTABILIZAÇÃO MENSAL DO PORTFOLIO 96

FIGURA 5.17 - SENSIBILIDADE SOBRE A TAXA DE DESCONTO 97 FIGURA 5.18 - SENSIBILIDADE SOBRE O PREÇO DO CONTRATO DE CURTO PRAZO 


\section{Lista de Tabelas}

TABELA 2-1 - DESPACHO RESULTANTE DA PRIMEIRA HORA 24

TABELA 2-2 - DESPACHO RESULTANTE DA SEGUNDA HORA 24

TABELA 2-3 - ESTRATÉGIA ALTERNATIVA DE OPERAÇÃO 25

TABELA 3-1 - EFEITO NO PREÇO DAS OPÇÕES 51

TABELA 5-1-CONTRATOS DE COMPRA 81

TABELA 5-2- CONTRATOS DE VENDA 81

TABELA 5-3- ESTATÍSTICAS DE VALOR ESPERADO E VALORES EM RISCO 86

TABELA 5-4- RISCO VOLUMÉTRICO 92

TABELA 5-5- VALOR PRESENTE DO PORTFOLIO 93

TABELA 5-6- VALOR DAS OPÇÕES (R\$MWH) 95 


\section{Lista de Abreviações}

$\begin{array}{ll}\text { ACL } & \text { - Ambiente de Contratação Livre } \\ \text { ACR } & \text { - Ambiente de Contratação Regulado } \\ \text { ANEEL } & \text { - Agência Nacional de Energia Elétrica } \\ \text { CCEE } & \text { - Câmara de Comercialização de Energia Elétrica } \\ \text { CMO } & \text { - Custo Marginal de Operação } \\ \text { ONS } & \text { - Operador Nacional do Sistema Elétrico } \\ \text { PLD } & \text { - Preço de Liquidação de Diferenças }\end{array}$

